\title{
Two stage ejector as a pre-stage of the water ring vacuum pump
}

\author{
Róbert Olšiak ${ }^{*}$, Marek Mlkvik, František Ridzoň, and Pavol Slovák \\ Slovak University of Technology, Faculty of M.E., Institute of Energy Machinery, Námestie Slobody 17, 812 31 Bratislava, \\ Slovakia
}

Received: 25 March 2019 / Accepted: 5 October 2019

\begin{abstract}
A supersonic gas ejector in conjunction with a liquid ring vacuum pump is used for creating and maintaining a vacuum in a chamber for technological purposes. In this paper, the authors submit an overview of the problematics of suction pressure reduction with a supersonic gas ejector used as a pre-stage of a liquid ring vacuum pump. This system has also the function of a cavitation protection due to the higher pressure present at the suction throat of the vacuum pump. A part of this paper is devoted to the governing equations used at the definition of the flow through an ejector. The CFD analysis of the problem was implemented with the package Fluent in 2 dimensions using the axisymmetric approach. The parts of the physical model were printed on a STRATASYS 3D printer, or were cast from technical resin. The experimental studies are then carried out in our own laboratory for validation purposes.
\end{abstract}

Keywords: Two stage ejector / liquid ring vacuum pump / cavitation

\section{Introduction}

The liquid ring vacuum pumps are technological devices used to create and maintain a vacuum for technological purposes. In practice, the vacuum means the state of a diluted gas, where the absolute pressure is lower than the atmospheric. Liquid ring vacuum pumps can be used at extraction of condensable vapor and gas mixtures. In this case, a part of the pumped mixture is condensable in the form of vapor inside the vacuum pump, while the condensate is drained with the working fluid. In other vacuum pump types, this phenomenon is undesirable due to the present lubricating grease. The relatively low efficiency and the high minimal suction pressure can be considered as a disadvantage. This minimal pressure is limited by the used working fluid inside the vacuum pump. It is problematic to determine this correlation because the parameters of the liquid ring vacuum pump are bounded not only to the type of used fluid (density, viscosity) but also to the change of these properties in dependence on the temperature and pressure. It is possible to determine the cavitation start by measuring of accompanying phenomena of cavitation, such as vibration or noise [1].

The barrier of the minimal suction pressure, at which the vacuum pump can be used, is depending on the fluid properties, construction type, number of stages, etc. According to the following authors, this pressure is stated as: Bannwarth [2] $5 \mathrm{kPa}$, Faragallah [3] $15 \mathrm{kPa}$. In practice,

\footnotetext{
* e-mail: robert.olsiak@stuba.sk
}

it is a common requirement to reach the suction pressure, which is much lower than the listed values. This is not possible due to the occurrence of cavitation inside the vacuum pump. This can be partially solved by lowering the pressure ratio, by using a multistage liquid ring vacuum pump, however even this solution is unacceptable from the viewpoint of a long-term run. The suction pressure can be lowered using a gas ejector as a pre-stage of the vacuum pump. This arrangement can reduce the pressure up to $1 / 5$ of the initial pressure.

\section{The options of the suction pressure reduction}

The ejector, as a technological device, is widely used in different applications, due to its simple construction and operational reliability. Basically, it is a device, in which the primary medium entrains the secondary driven medium and accelerates it to the supersonic speed at reduced pressure. The pressure measured at the ejector inlet $\mathrm{p}_{2}$ is decisive to achieve the design pressure in the considered pressure chamber. From the viewpoint of ejector design, there are some limiting parameters (Fig. 1): pressure measured at the ejector outlet $\mathrm{p}_{3}$ and the mass flow of the mixture $\dot{m}_{3}$, which are corresponding to the occurrence of cavitation inside the vacuum pump. The values of the primary (driving) fluid $p_{1}$ and $\dot{m}_{1}$ are used at the design of the primary supersonic nozzle. In case that the ejector is correctly designed, it will extend the working range of the liquid ring vacuum pump as seen in Figure 1. 

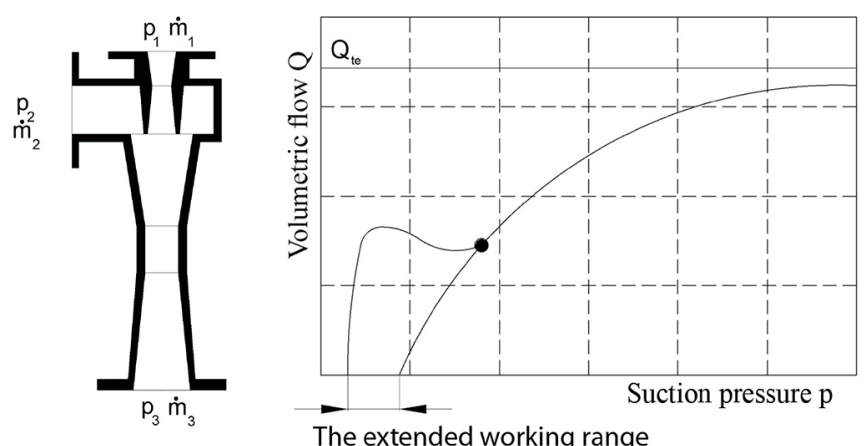

Fig. 1. Construction of a supersonic ejector (left) and performance characteristics of a liquid ring vacuum pump with and without a supersonic ejector pre-stage.

From this simple analysis it is apparent, that for the correct design of a supersonic ejector, it is necessary to know the following parameters:

- the required minimal suction pressure $p_{2}$ at the chamber throat;

- the required mixture mass flow $\dot{m}_{2}$ at the chamber throat;

- the minimal suction pressure $p_{3}$ of the vacuum source.

It is obvious, that the pressure $p_{2}$ and mass flow $\dot{m}_{2}$ are the chosen parameters of the designed ejector. The suction pressure $p_{3}$ and mass flow $\dot{m}_{3}$ can be specified either experimentally, which is the most commonly used method, or with theoretical calculation according to the geometrical dimensions of the device, operational data, and properties of the working fluid. The determination of the ability of air, or gas, extraction from a chamber is carried out through the analysis of performance characteristics of the vacuum system. This can be defined as the effective flow rate of the pumped air measured at the given suction pressure (1):

$$
Q_{E}=f\left(p_{s}\right) .
$$

\section{The governing equations}

The mostly used ejector design method is based on a 1-dimensional analysis of compressible gas flow. This method was first proposed by Keenan (1942) and Neumann (1950) and it uses the following assumptions:

- the kinetic energy on the inlet of both fluids is negligible;

- the two working fluids are considered as ideal gases;

- at the end of the mixing chamber, both fluids are uniformly mixed, without occurring shocks inside the mixing chamber.

Based on these assumptions, equations (2)-(4) describe the character of the flow from the inlets to the outlet [4]: - the momentum equation

$$
p_{1} A_{1}+\rho_{1} Q_{1} v_{1}+p_{2} A_{2}+\rho_{2} Q_{2} v_{2}=p_{3} A_{3}+\rho_{3} Q_{3} v_{3},
$$

- the continuity equation

$$
\rho_{1} Q_{1}+\rho_{2} Q_{2}=\rho_{3} Q_{3}
$$

- the energy equation for adiabatic flow

$$
\dot{m}_{1}\left(h_{1}+\frac{v_{1}^{2}}{2}\right)+\dot{m}_{2}\left(h_{2}+\frac{v_{2}^{2}}{2}\right)=\dot{m}_{3}\left(h_{3}+\frac{v_{3}^{2}}{2}\right) .
$$

The required compression ratio $(\mathrm{CR})$ of an ejector determines the ratio of the static pressures on the ejector exit (identical to the pressure measured at the inlet throat of the vacuum pump) to the pressure measured at the secondary suction throat of the ejector (5). The entrainment ratio (ER) [5] expresses the relationship between the desired mass flow rates through the secondary and primary inlets of the ejector.

$$
C R=\frac{p_{3}}{p_{2}}, E R=\frac{\dot{m}_{2}}{\dot{m}_{1}}=\frac{p_{2}}{p_{1}} \frac{A_{2}}{A_{1}}\left(\frac{T_{1}}{T_{2}}\right)^{\frac{1}{2}} \frac{f_{a}\left(\kappa_{2}, M_{2}\right)}{f_{b}\left(\kappa_{1}, M_{1}\right)},
$$

where

$$
f(\kappa, M)=\frac{\dot{m}}{p A}\left(R T_{0}\right)^{\frac{1}{2}}=M\left[\kappa\left(1+\frac{\kappa-1}{2} M^{2}\right)\right]^{\frac{1}{2}} .
$$

\section{Design methods}

The motion of the working fluid is given by the pressure difference between the atmospheric air, at the primary inlet, and the minimal suction pressure given by the vacuum pump. Due to the small molecular weight of the used working fluid (atmospheric air), the value of the kinetic energy of the primary fluid depends on the outlet speed of the supersonic nozzle. For this reason, the nozzle is considered as the most important part of an ejector. The mostly used nozzle geometry due to its simple design is conical, with constant wall angle $\left(12^{\circ}-18^{\circ}\right)$. Since the outlet velocity field has also a radial component, it is necessary to introduce a correction factor, which takes into account the ratio of the ideal momentum of the conical nozzle, with the axial component of the velocity.

Parabolic de Laval nozzles take into account the presence of Prandtl-Mayer expansion [6]. The shape of the nozzle is obtained either by an empirical method, or by the method of characteristics (MoC) (Fig. 2).

The Prandtl-Meyer formula (6) is used to determine the Mach number at a given point of the flow field followed by the Mach angle calculation (7).

$$
\begin{aligned}
v(M) & =\sqrt{\frac{\kappa+1}{\kappa-1}} \arctan \sqrt{\frac{\kappa-1}{\kappa+1}\left(M^{2}-1\right)} \\
& -\arctan \sqrt{M^{2}-1}
\end{aligned}
$$

$$
\mu=\arctan \frac{1}{M} .
$$

These equations alongside with the equations of quasi one-dimensional-isentropic flow of compressible fluids in ducts have been automated and software was developed 
$[5,7]$. The flowchart of the calculation can be seen in Figure 3 .

The developed design software allows determining the exact shape of the desired nozzle type with the option of exporting the generated coordinates in common formats. The software can also be used to apply the numeric simulation of the flow in the nozzle via Mac Cormack method. Figure 4 compares the progress of static pressure along the axis of the supersonic nozzles with variable inlet pressures. According to the theory of the quasi 1D flow the critical pressure $p^{*}$ is relative to the smallest cross-section area and depends only on the value of the inlet pressure, in case that the compression ratio is constant $(\mathrm{CR}=23.2)$.

The high-speed mixture of the primary air with secondary working fluid is entering the mixing chamber. The 1D design model uses the constant-area mixing model,



Fig. 2. Divergent portion of a parabolic de Laval nozzle with characteristic lines and distribution of the observed parameters (left), the velocity components with the angles used by the method of characteristics. for which the supersonic nozzle exit is located directly on the cross-section plane of the mixing chamber inlet (Fig. 5).

With use of the ER alongside with equation (1) and the known flow rate at the diffuser outlet, the velocity at the exit of the mixing chamber can be obtained:

$$
v_{\text {mch,ex }}=\eta_{\text {mixing }}\left(\frac{\dot{m}_{1} v_{1}+\dot{m}_{2} v_{2}}{\dot{m}_{1}+\dot{m}_{2}}\right) .
$$

\section{Experimental model of the two-stage ejector}

On the basis of the governing equations [8], the quasi 1D assumption, and semi-empirical relationships a physical model of a two-stage integrated supersonic ejector was constructed. Since the high momentum of the driving air is achieved by high velocity magnitude at the primary nozzle discharge, the following distributions of driven and driving mass flows were chosen (Fig. 6):

The following factors were taken into account at the design process:

- The manufacturability of the parts: avoiding extremely small diameters and lengths.

- The discharge velocity of the II. stage shall be equivalent to the velocity measured at the suction throat of the vacuum pump.

- Location of the pressure sensors should not negatively affect the measured parameters.

- Replaceable of the I. stage nozzles.

- Mechanical stability of the integrated ejector.

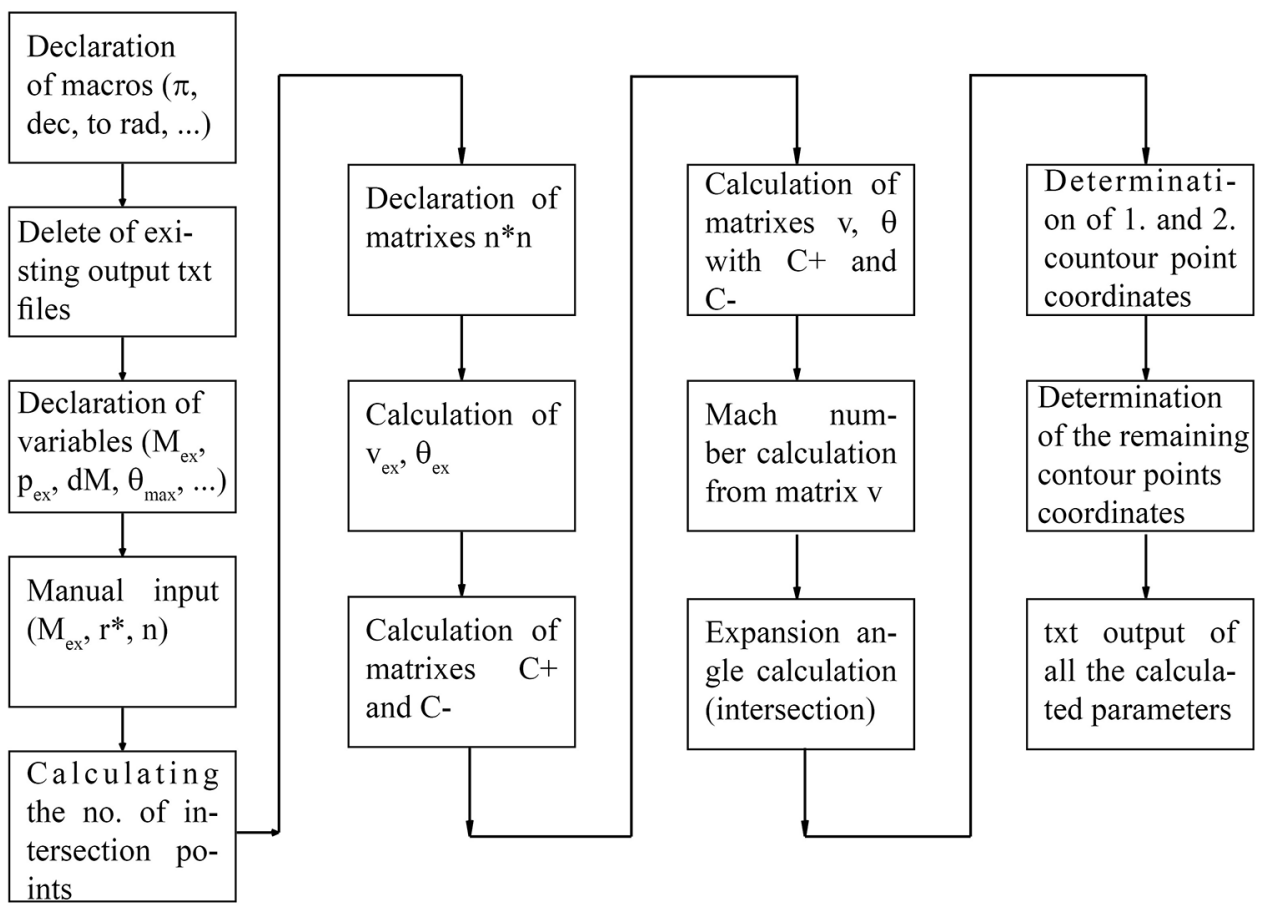

Fig. 3. Flowchart of the automated calculation process. 


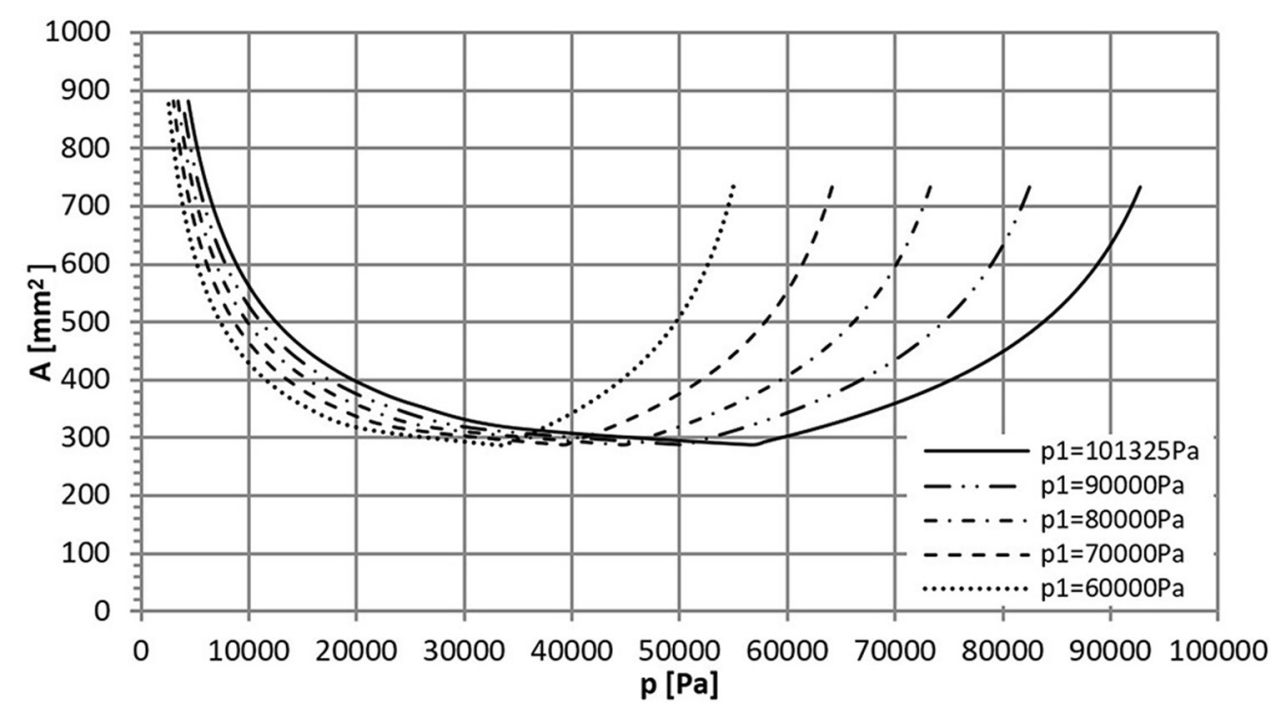

Fig. 4. Influence of the inlet pressure $p_{1}$ on the discharge pressure of the supersonic nozzle.


Fig. 7. 3D model of the designed two-stage ejector.

Fig. 5. Constant-area-mixing chamber.



Fig. 6. Distribution of the mass flow between the I. and II. ejector stage.

Subsequently, a 3D two-stage ejector model was created in the CAD software CATIA (Fig. 7).

Some parts were cast from technical dentacryl, then machined on lathe machines. The next parts were printed on a STRATASYS 3D printer, then glued together as needed. The following (Fig. 8) shows 3 interchangeable supersonic nozzle types used at the I. stage.

The first two nozzles (a) and (b) were printed without correcting the internal dimension errors made due to the resolution and accuracy of the $3 \mathrm{D}$ printing. In order to correct the inaccuracy of the printing process the nozzle type (c) was designed, which has a slightly reduced critical diameter and a $10 \%$ increase of the discharge diameter. The following (Fig. 9) shows a close-up detail at the inlet portion of the I. ejector stage.

The middle part connecting the two ejector stages including the supersonic nozzle and the inlet tube was also created using a $3 \mathrm{D}$ printer. To avoid the air leakage and the mixing of the stream discharged through the supersonic nozzle with the air around the inlet tubes, a two-part design was chosen. The dividing plane (red line) and the geometry are shown in Figure 10.

\section{The CFD analysis}

This analysis was implemented with the CFD package Fluent in 2 dimensions using the axisymmetric approach. Due to the rapid change in density and other thermodynamic parameters in the flow field, the density based solver was chosen. The small gaps in the geometry, mainly at the critical cross-section area of both supersonic nozzles, require a denser mesh to realistically predict the change of the observed parameters at these places. The main parts of the ejector were analyzed separately using settings listed in Table 1. 

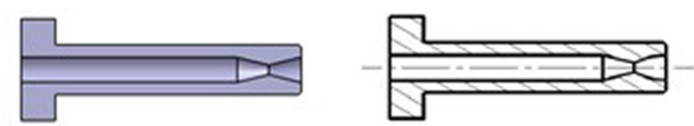

a)


b)
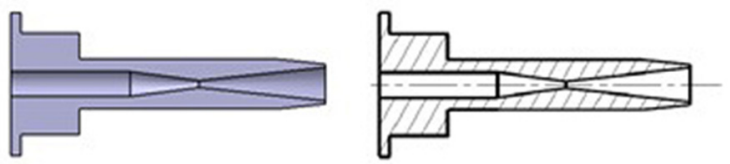

c)

Fig. 8. Various supersonic nozzle designs of the I. Stage.


Fig. 9. Detail of the inlet portion of the I. ejector stage. Legend: 1-supersonic nozzle, 2-silicone sealant, 3-nozzle holder, 4-inlet tube, 5-mixing chamber.

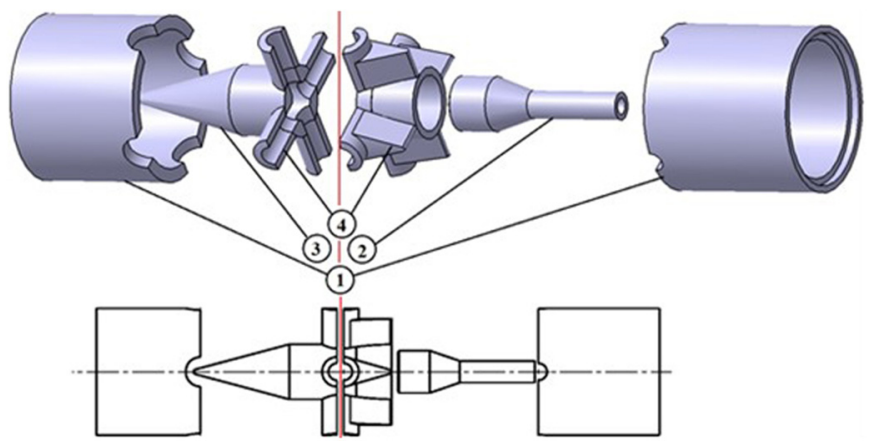

Fig. 10. Detail of the middle section of the two-stage ejector. Legend: 1-casing, 2-supersonic nozzle, 3-cone, 4-aerodynamic inlet tubes.

As the most important part of the ejector, at first, the supersonic nozzle of the II. stage was simulated. After the discharge cross-section area of the nozzle (at radius $r_{\mathrm{ex}}$ ) a rectangular area (in 2D) was connected with dimensions $2 r_{\text {ex }} \times 22 r_{\text {ex }}$, Table 2 .

Figure 11 represents the above described case, with the defined boundary types. The pressure inlet on the left side is defined by the gauge and the total pressure of $101325 \mathrm{~Pa}$ at ambient temperature of $293.15 \mathrm{~K}$. The other ambient conditions were re-calculated using real gas equation.

The gradually increasing backpressure from the nominal design pressure $(2520 \mathrm{~Pa})$ resulted in a decrease of the outlet velocity and hence the adiabatic efficiency of the nozzle.

Table 1. Global setting of the CFD case (valid in all CFD simulations).

\begin{tabular}{lll} 
Mesher & Max. cell size: & $0.1 \mathrm{~mm}$ \\
& Size function: & Proximity \\
Cell type: & Quad \\
Boundary layer & True \\
Boundary layer setting: & Smooth transition \\
Transition ratio & 0.6 \\
& Max. layer count & 25 \\
Fluent & Solver & Density based \\
& Dimension & $2 \mathrm{D}(x-y)$ \\
& Precision & Double \\
& Velocity formulation & Absolute \\
& Gradient & Green-Gauss Cell \\
& Calculation scheme & Implicit \\
& Time & Steady state \\
Energy equation & True \\
Turbulence modeling & $k$ - $\omega$ SST \\
Medium & Real gas \\
Gas equation & Soawe-Redlich-Kwong \\
\hline
\end{tabular}

Table 2. Geometry of the supersonic nozzle of the II. stage with the connected rectangular area.

Confusser length

Diffuser length

Length of the connected area

Inlet diameter of the nozzle

Critical diameter of the nozzle

Outlet diameter of the nozzle

Diameter of the connected area

\begin{tabular}{ll}
$L_{\mathrm{konf}}$ & $30.40 \mathrm{~mm}$ \\
$L_{\mathrm{n}, \mathrm{dif}}$ & $4.71 \mathrm{~mm}$ \\
$L_{\text {exp }, p}$ & $76.56 \mathrm{~mm}$ \\
$d_{1}$ & $16.54 \mathrm{~mm}$ \\
$d^{*}$ & $1.45 \mathrm{~mm}$ \\
$d_{\text {ex }}$ & $3.48 \mathrm{~mm}$ \\
$d_{\exp , n}$ & $6.96 \mathrm{~mm}$ \\
\hline
\end{tabular}

As can be seen in Figure 12, the relatively small change in the back pressure does not affect the flow field in the rectangular area and inside the nozzle.

The course of the static pressure monitored alongside the nozzle axis is compared depending on the back pressure present (Fig. 13). The marked point $\mathrm{F}$ is located $13.89 \mathrm{~mm}$ from the nozzle discharge cross-section area, so even a $7.9 \times$ increase of the back pressure has no effect on the flow stability inside the nozzle.

The aim of the next analysis was a $2 \mathrm{D}$ model of a twostage ejector with the following parameters of the driving medium at both stages $p_{\mathrm{I}, 01}=p_{\mathrm{II}, 01}=101 \quad 325 \mathrm{~Pa}$, $T_{\mathrm{I}, 01}=T_{\mathrm{II}, 01}=293.15 \mathrm{~K}$. The properties of the driven air are $m_{\mathrm{I}, 02}=0 \mathrm{~kg} / \mathrm{s}, p_{\mathrm{I}, 02}=2493 \mathrm{~Pa}, T_{\mathrm{I}, 02}=293.15 \mathrm{~K}$. To increase the convergence rate of the calculation, the following procedure was applied:

- the CFD model was initialized for the flow of ideal gas, without modeling turbulent phenomena;

- when the 400-500 iterative steps were reached, the k- $\omega$ SST turbulence model was switched on; 



Fig. 11. Mesh of the supersonic nozzle with the connected rectangular area.



Fig. 12. Contours of the Mach number at various back pressures.

- after the monitored mass flow stabilized at the output of the two-stage ejector, the gas equation was switched to real gas (Fig. 14).

In this way, convergence was achieved about up to 2000 iterative steps. On the basis of the static pressure contours shown, it is clear that the I. ejector stage nozzle operates with a twice lower output pressure (from $p_{\text {atm }}$ to $2583 \mathrm{~Pa}$ ) than the II. stage (5128 Pa), which means that considerable differences $\left(671 \mathrm{~m} \mathrm{~s}^{-1}\right.$ and $\left.548 \mathrm{~m} \mathrm{~s}^{-1}\right)$ will also be noticeable at output speeds.

\section{The experimental setup}

The experimental part consists of the measurement of the performance characteristics $Q_{\mathrm{E}}=f\left(p_{\mathrm{s}}\right)$ of the vacuum pump type SZO-65 with the two-stage ejector (Fig. 15). The ejector stages are connected in series, so the outlet of the first stage is connected to the secondary suction of the second stage. During the experiments, an effort was made to maintain constant physical conditions (ambient temperature and pressure, RPM), especially maintaining the temperature of the coolant/working fluid close to $20^{\circ} \mathrm{C}$, where the critical pressure, at which the cavitation occurs, is $2338 \mathrm{~Pa}$.

The experimental circuit meets the following assumptions and criteria:

- The suction pressure sensor is located as close as possible to the suction throat of the vacuum pump.

- Smooth regulation of the suction pressure enabled through the shut-off valve. 


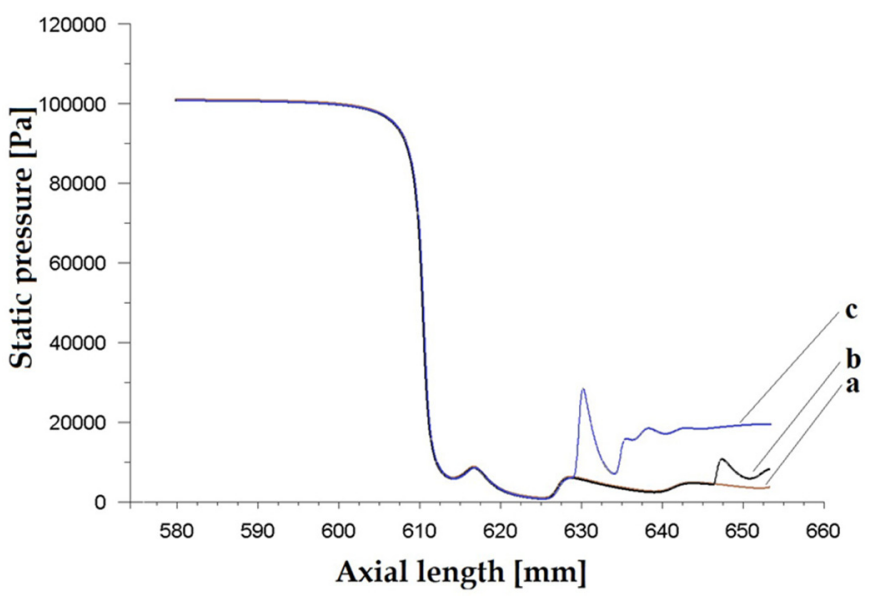

Fig. 13. Courses of the static pressure monitored alongside the nozzle axis at various back pressures.



Fig. 15. Dependency of the saturated vapor pressure on the fluid temperature.

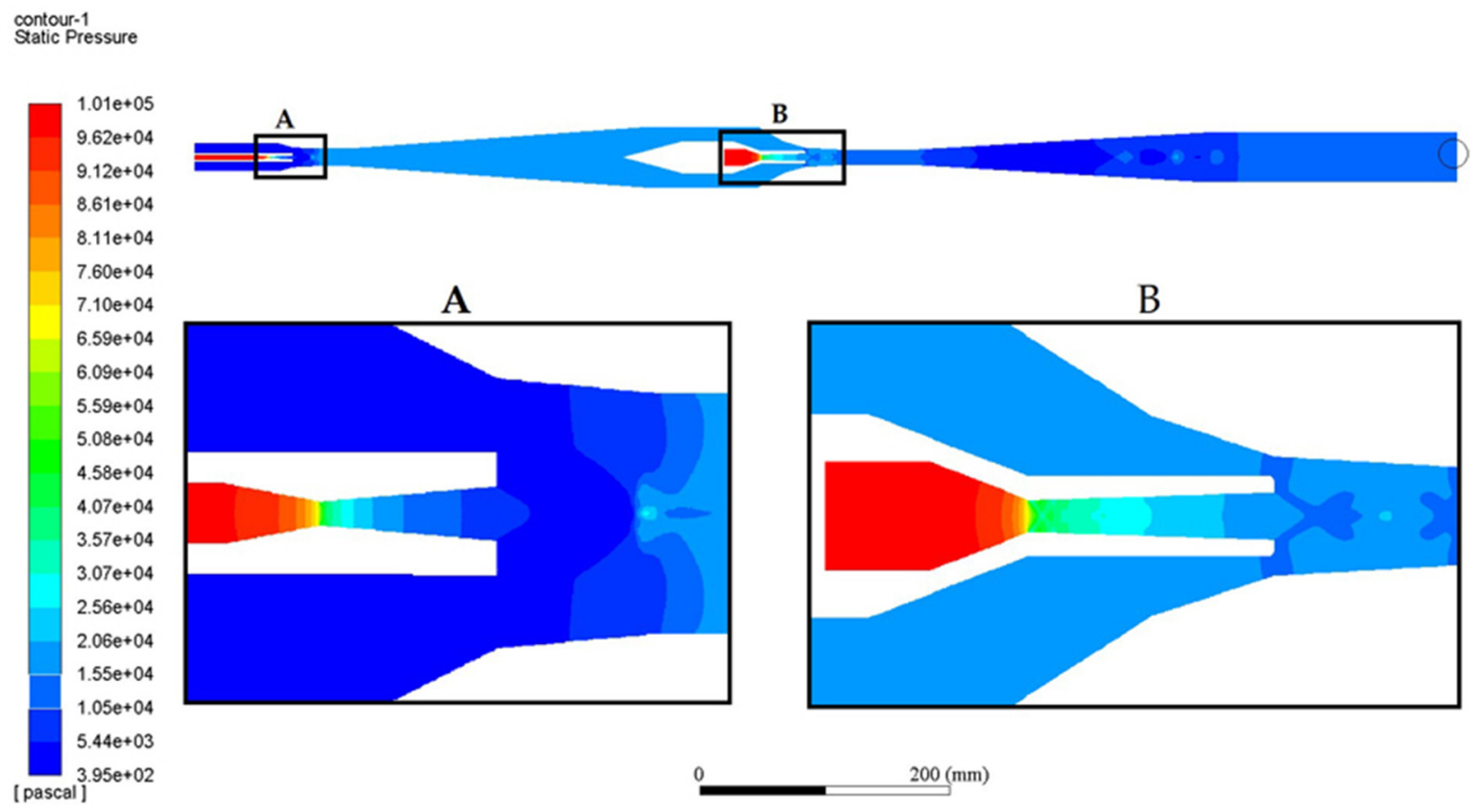

Fig. 14. Contours of the static pressure with details of the supersonic nozzles of both stages.

- The hydraulic part of the circuit contains minimal amount of local loss factors (bends, reductions, ...) that could distort the values of the measured parameters.

- The used sensors are calibrated according to the higher order reference sensors.

- The working fluid is supplied from a sufficiently large tank that allows the water to circulate without heat accumulation.

The experimental setup is shown in the following figure (Fig. 16):

For the measurement of the non-electric parameters the following sensors were used (Fig. 17):

- Diaphragm pressure transmitter BD-SENSORS DMP $331 \mathrm{i}$ with range of 0 to $4 \mathrm{kPa}$ absolute pressure, 3-wire version with $0-10 \mathrm{~V}$ output signal voltage.
- Differential pressure sensor BD-SENSORS DPS 200 with range of 0 to $10 \mathrm{kPa}, 3$-wire version with $0-10 \mathrm{~V}$ output signal voltage.

- Laminar flowmeter OMEGA FMA-1600 A with range of 0 to 3000 SLPM.

- Laser tachometer.

The experiment begins by running the pump of the external circuit to constantly deliver water to the pump. As the housing of the vacuum pump fills up to the desired level, the operating speed 1450 RPM was set with the frequency converter. The shut-off valve located on the motive fluid inlet of both stages was fully opened. After the stabilization of the monitored values, the first point of the $Q_{E^{-}} p_{s}$ diagram was recorded. By gradually closing the valve on the secondary inlet port the suction pressure was reduced. At each stop, the actual point data was recorded. The physical 




Fig. 16. Experimental setup. Legend: SV - shut-off valve, $\mathrm{M}$ - 3-phase asynchronous electric motor, VV - water-ring vacuum pump, SEP - separator, OC - circulating pump, N tank, PR - transducer, PC - measuring computer, QE [SLPM] flow rate, $t_{\mathrm{A}}\left[{ }^{\circ} \mathrm{C}\right]$ - temperature at vacuum pump inlet port, $\mathrm{t}_{B}\left[{ }^{\circ} \mathrm{C}\right]$ - temperature at separator inlet port, $p_{\mathrm{s}}[\mathrm{Pa}]-$ suction pressure, $M_{\mathrm{k}}[\mathrm{Nm}]$ - torque, $t_{\mathrm{atm}}\left[{ }^{\circ} \mathrm{C}\right]$ - ambient temperature, $p_{\text {atm }}$ $[\mathrm{Pa}]$ - atmospheric temperature, $H_{\text {atm,rel }}[\%]$ - relative humidity.
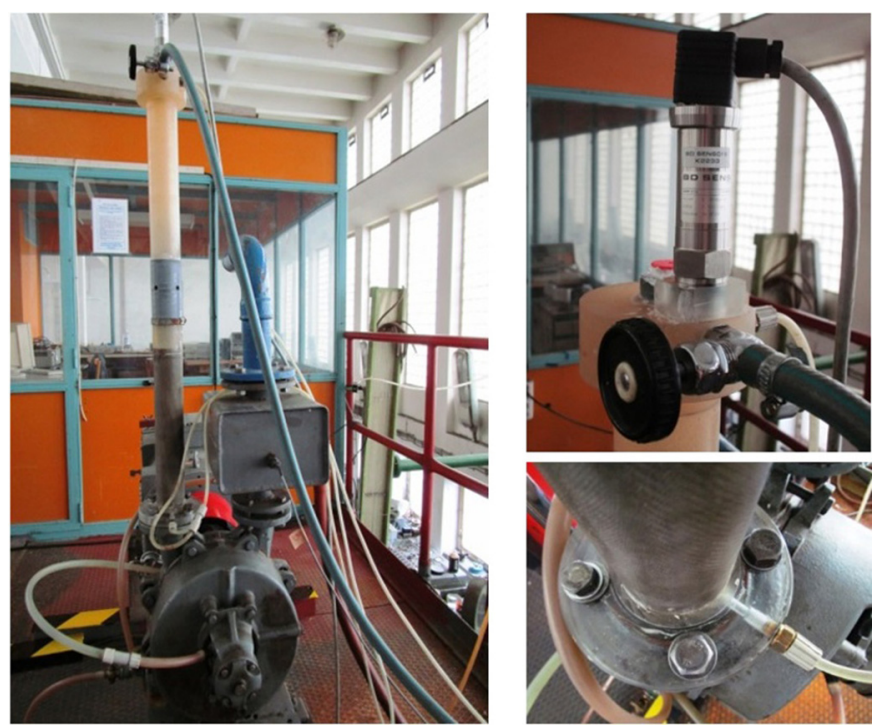

Fig. 17. Experimental model of the two-stage integrated ejector (left), pressure sensor DMP 331i (right up), static pressure measuring point at the suction throat of the vacuum pump (right down).

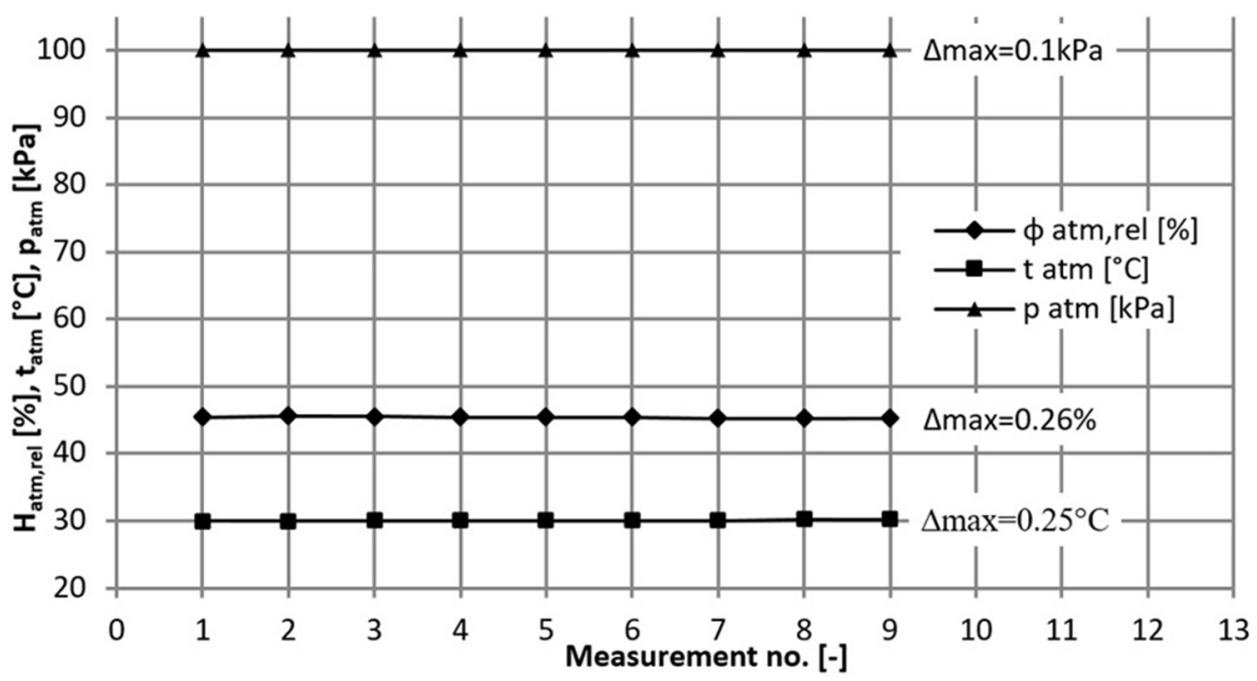

Fig. 18. Progress of the ambient physical parameters during the experiment.

parameters of ambient air during the measurement are shown in Figure 18.

The measured $Q_{E}-p_{s}$ values were imported to NI Diadem software and then the characteristics were plotted as shown in Figure 19. According to the theory of PrandtlMeyer flow, the MoC nozzle should convert the available pressure drop, given between the inlet and the discharge of the nozzle, into kinetic - motive energy more efficiently, but due to the inaccurate printing process, the output parameters have deteriorated.

Figure 20 combines the performance characteristics of the water-ring vacuum pump itself and the twostage ejector mounted. During the operation, the ejector extends the lower vacuum boundary, fulfilling the function of cavitation protection of the vacuum pump.

To measure the change in suction pressure and flow rate at successive $1450 \mathrm{RPM}$, the behavior of ejector was monitored at other than nominal revolutions per minute. At the first measurement, a zero mass flow rate of the driven medium on the stage I. was set. The ejector at a set $1200 \mathrm{RPM}$ achieves suction pressure at $4753 \mathrm{~Pa}$, and $14170 \mathrm{~Pa}$ was measured at the suction throat of the vacuum-pump $\left(\mathrm{CR}_{\mathrm{I}-\mathrm{II}}=2.98\right)$. It can be stated that the suction pressure measured at 1400 RPM approaches the design value. At $1500 \mathrm{RPM}$ a slight reduction in suction pressure is observed over a design value. Figure 20 presents the experimental results at various rotation speeds. 


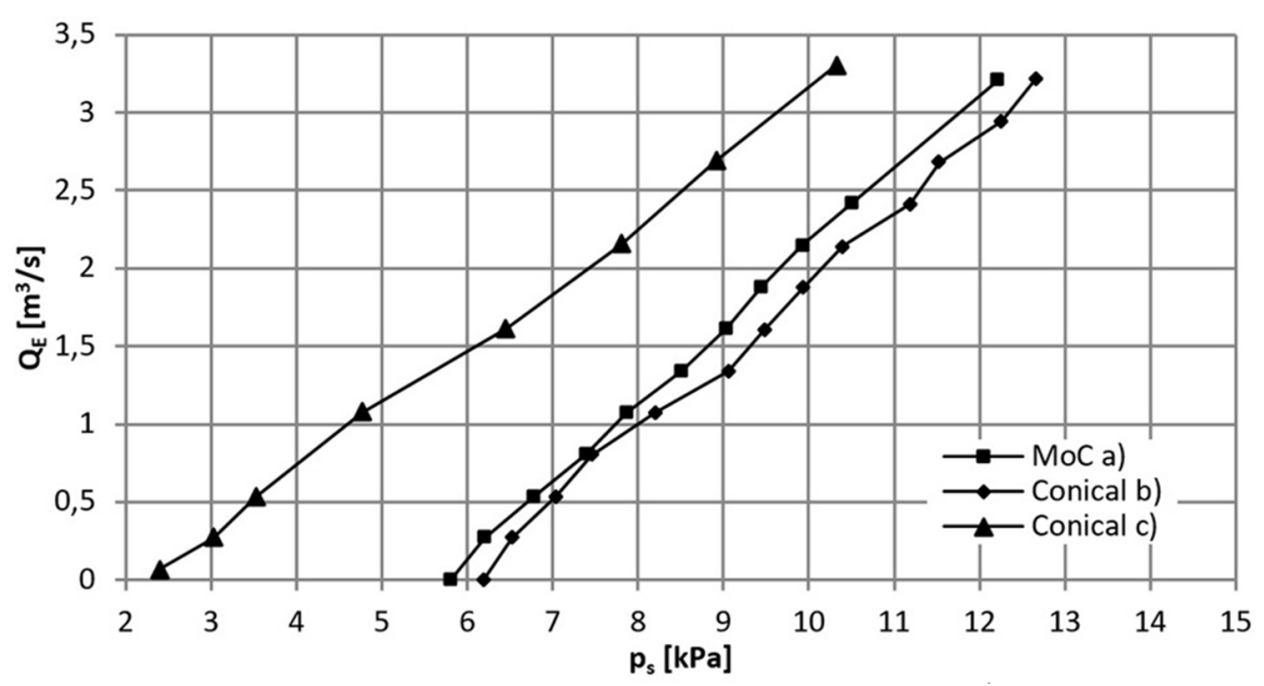

Fig. 19. Progress of the ambient physical parameters during the experiment.

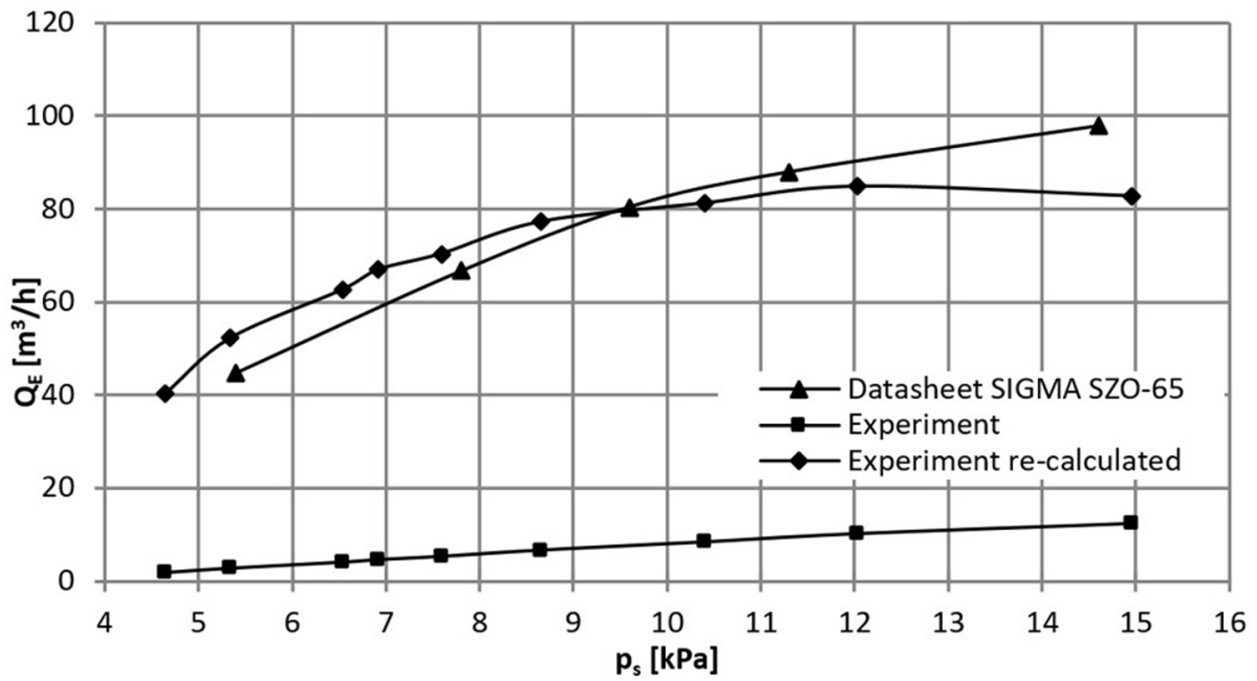

Fig. 20. Performance characteristics of the water-ring vacuum pump and the two-stage ejector (re-calculated values are corrected by the suction pressure).

\section{The comparison of results and conclusion}

In this section, we compare the results obtained from the numerical modeling of flow with the developed design software, the results of CFD simulation, and experimental measurements on the physical model of a two-stage ejector (Fig. 21). In comparison, 3 quantitative criteria are used: ejector suction pressure $p_{s}=p_{\mathrm{I}, 02}$, volume flow $Q_{E}$ and suction pressure $p_{\mathrm{vv}}=p_{\mathrm{II}, 04}$. The comparison of the monitored parameters in the internal nodes of the ejector is possible only for the results of CFD calculations and for the output values from the design software since only 3 variables $\left(p_{\mathrm{vv}}, p_{s}, Q_{E}\right)$ were measured during the experiments, Table 3.

Based on these results, we can state the following:
Ejector designed by 1D method and semi-empirical relationships should work at compression ratio:

$$
\mathrm{CR}_{I-I I \rightarrow \text { software }}=\frac{14960 \mathrm{~Pa}}{2493 \mathrm{~Pa}}=6
$$

which is the operation in the design state. However, based on experimental results valid for the optimized cone nozzle, the maximum compression ratio achieved with the closed shut-off valve is:

$$
\pi_{I-I I, \text { experiment }}=\frac{10080 \mathrm{~Pa}}{2400 \mathrm{~Pa}}=4.2 .
$$

The ejector fulfills its function of reducing the system's final vacuum value from the original $4650 \mathrm{~Pa}$ to $2400 \mathrm{~Pa}$ at 


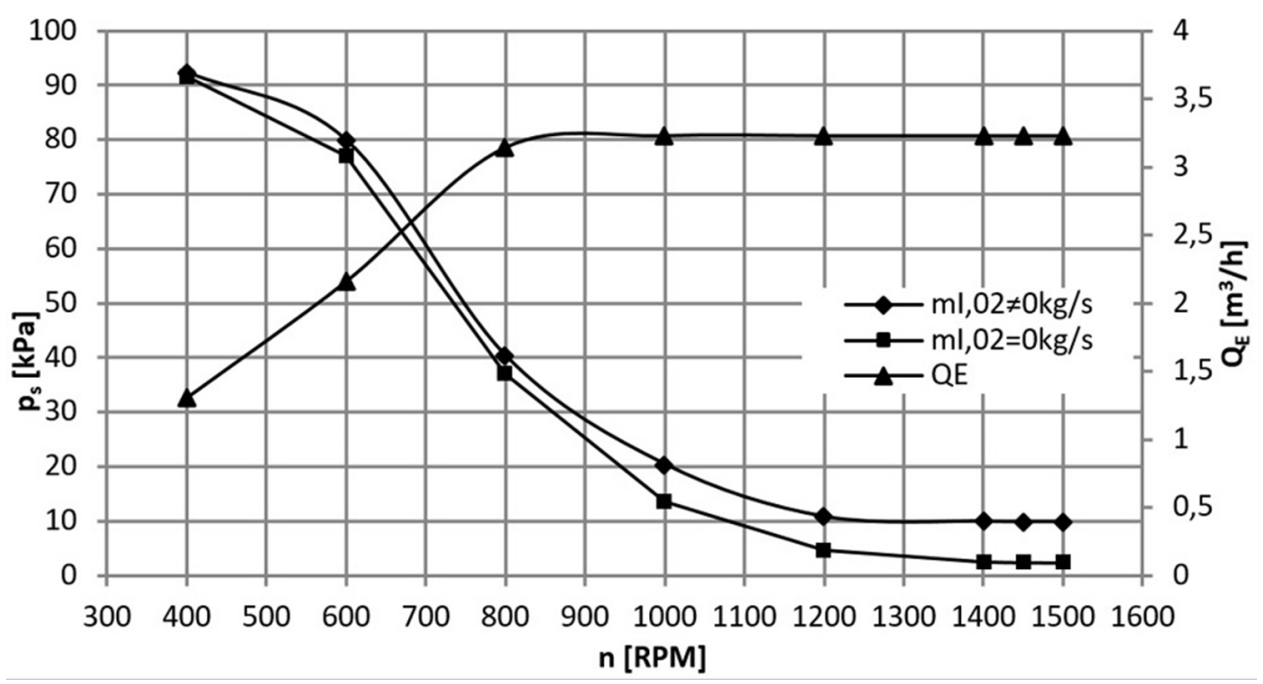

Fig. 21. RPM characteristic of the two-stage ejector at opened and partially closed shut-off valve.

Table 3. Comparison of the results obtained from the design software, CFD simulation and from 3 experimental measurements.

\begin{tabular}{llll}
\hline Source of the data & $\begin{array}{l}p_{s}=p_{\mathrm{I}, 02} \\
{[\mathrm{~Pa}]}\end{array}$ & $\begin{array}{l}Q_{E} \\
{\left[\mathrm{~m}^{3} / \mathrm{h}\right]}\end{array}$ & $\begin{array}{l}p_{\mathrm{vv}}=p_{\mathrm{II}, 04} \\
{[\mathrm{~Pa}]}\end{array}$ \\
\hline Design software & 2493.00 & 1.06 & 14960.00 \\
CFD - ANSYS & 2557.00 & 0.00 & 15522.00 \\
Experiment - conical nozzle & 6190.00 & 0.00 & 13370.00 \\
Experiment - MoC nozzle & 5810.00 & 0.00 & 13130.00 \\
Experiment - optimized conical nozzle & 2400.00 & 0.00 & 10080.00 \\
\hline
\end{tabular}

zero airflow. Running the pump itself without using the ejector at a given working fluid temperature would mean operation in a cavitation mode. After connecting the ejector, the suction pressure measured on the pump suction throat was reduced by $4880 \mathrm{~Pa}$, so the pump and ejector assembly did not work in the design state, which may be caused by:

- inaccuracies occurring during the production of the ejector parts,

- leakage within the ejector,

- a measurement error of the performance characteristic of the water ring that served at the ejector design stage.

\section{Nomenclature}

A Cross section area, $\mathrm{m}^{2}$

$h \quad$ Enthalpy, $\mathrm{kJ} \mathrm{mol}^{-1}$

$M$ Mach number

$\dot{m}$ Mass flow, $\mathrm{kg} \mathrm{s}^{-1}$

$p$ Pressure (absolute), $\mathrm{Pa}$

$Q$ Volumetric flow, $\mathrm{m}^{3} \mathrm{~h}^{-1}$

$R \quad$ Universal gas constant, $\mathrm{J} \mathrm{K}^{-1} \mathrm{~mol}^{-1}$

$r$ Radius, mm

$t$ Temperature, ${ }^{\circ} \mathrm{C}$

$T$ Temperature $\mathrm{K}$ $v \quad$ Velocity, $\mathrm{m} \mathrm{s}^{-1}$

Adiabatic exponent

$\eta \quad$ Mixing ratio

Acknowledgments. This contribution was created on the basis of the project "Research centre ALLEGRO" (ITMS project code: 26220220198), supported by Operational Programme Research and Development funded by the European Regional Development Fund. The authors gratefully acknowledge the contribution of the Scientific Grant Agency of the Slovak Republic under the grant VEGA 1/0743/18.

\section{References}

[1] J. Jablonská, M. Mahdal, M. Kozubková, Spectral analysis of pressure, noise and vibration velocity measurement in cavitation, J. Inst. Meas. Sci. Slovak Acad. Sci. 17, 250 $256(2017)$

[2] A. Roth, Vacuum technology, Elsevier Science Publishing, Amsterdam, New York, 1982

[3] M. Wutz, H. Adam, W. Walcher, Theorie und Praxis der Vakuumtechnik, Friedr. Vieweg and Sohn Verlagsgesellschaft, Braunschweig/Wiesbaden, 1992

[4] Ch. Liao, Gas ejector modeling, Theses, Texas A\&M University, TX, 2008 
[5] M. Puškár, T. Brestovič, N. Jasminská, Numerical simulation and experimental analysis of acoustic wave influences on brake mean eff. pressure in thrust-ejector inlet pipe of combustion engine, Int. J. Veh. Des. 67, 63-76 (2015)

[6] M. Boun-jad, T. Zebbiche, A. Allali, High temperature gas effect on the Prandtl-Meyer function with application for supersonic nozzle design, Mech. Ind. 18, 9 (2017)
[7] R. Olšiak, Z. Fuszko, Z. Csuka, Reduction of the suction pressure of a liquid ring vacuum pump with a supersonic gas ejector, MATEC Web Conf. 168, 6 (2018)

[8] S. Daneshmand, C. Aghanajafi, A. Bahrami, Analytical and experimental methods of design for supersonic two-stage ejectors, Int. J. Aerospace Mech. Eng. 2, 141144 (2009)

Cite this article as: R. Olšiak, M. Mlkvik, F. Ridzon, P. Slovák, Two stage ejector as a pre-stage of the water ring vacuum pump, Mechanics \& Industry 20, 703 (2019) 